\title{
Consideraciones para la Comprensión de la Reproducción Social del Campesino Cubano: acercamiento desde la producción científica rural ${ }^{1}$
}

\author{
Yisel Herrera Martínez²
}

\begin{abstract}
Resumen: La reproducción del campesinado, unidad económica familiarclase social, se ha convertido en una preocupación mundial. La emergencia alimentaria y la necesidad de formas de producción sostenibles han catapultado a la agricultura familiar como alternativa de solución, por esta razón la actividad científica invierte en el estudio de la diversidad de las formas familiares y en los cambios en las estrategias de vida y reproducción social. El artículo que se presenta constituye una valoración acerca de los factores que intervienen en la reproducción del campesinado en Cuba, fruto de la reconstrucción teórica lograda desde la sociología del conocimiento. Esta perspectiva teórico-metodológica, establece la relación dialéctica entre el conocimiento científico que se ha producido y su contexto social de referencia; describe el contexto de implementación de las políticas económicas y las transformaciones sociales que refleja la ciencia acerca del medio rural. El resultado fundamental radica en la determinación de las formas de manifestación del capital económico, cultural y social, resultado de la interpretación de datos a partir del análisis de contenido y que fundamentan, desde una perspectiva histórico-social, la comprensión del campesinado y su reproducción como objeto de estudio de la ciencia en Cuba.
\end{abstract}

Palabras-claves: Campesinado; Reproducción social; Capital económico; Capital cultural; Capital social.

Abstract: The reproduction of the peasantry, as a family-economic unit and social class, has become a global concern. The food emergency and the need for sustainable forms of production have pushed up the family farming as an alternative solution; for this reason, the scientific activity invests in studying the diversity of family forms and changes in livelihood strategies and social reproduction. The article presented is a review about the factors involved in the reproduction of the peasantry in Cuba, result of theoretical reconstruction achieved from the sociology of knowledge. This theoretical and methodological perspective establishes the dialectical relationship between scientific knowledge that has

1. Data de submissão: 4 de junho de 2014. Data de aceite: 20 de agosto de 2015.

2. Centro de Estudios Socioculturales de la Universidad de Cienfuegos, Cienfuegos, Cuba E-mail: yhmartinez@ucf.edu.cu 
occurred, and the social context of reference; also describes the context of implementation of economic policies and social transformations that reflects the science about the countryside. The main result lies in determining the forms of manifestation of economic, cultural and social capital, which is the result of data's interpretation using content analysis and that support from a socio-historical perspective, understanding the peasantry and their reproduction, as a case study for science in Cuba.

Key-words: Peasantry; Social reproduction; Economic capital; Cultural capital; Social capital.

DOI - http://dx.doi.org/10.1590/1234-56781806-9479005303006

Classificação JEL: Z13.

\section{Introducción}

El reconocimiento de los capitales específicos que intervienen en la reproducción social del campesinado constituye un tema de significación, dada la importancia que reviste la caracterización de los diversos sujetos que participan en la producción de alimentos y el problema agrario en las sociedades en desarrollo. La crisis y el debate acerca de la soberanía alimentaria invitan a la comunidad científica y académica a descubrir alternativas para enfrentar estos retos. Para las ciencias sociales el reto radica, entre otras perspectivas, a realizar análisis histórico-sociales.

El presente artículo analiza desde este punto de vista, la producción científica desarrollada en Cuba después del triunfo de la Revolución para de identificar las particularidades de los capitales específicos que posee el campesinado y la posibilidad de realizar futuras investigaciones acerca de la reproducción social.

Definir al campesinado cubano resulta complejo, por el insuficiente tratamiento teórico como clase social en el contexto rural cubano;

3. En el caso cubano se distingue la ausencia de una teoría de la economía campesina diseñada sobre la base de las particularidades de la transición socialista en las condiciones de mundialización capitalista. Por esta razón la ciencia se apoya en las contribuciones existentes en el campo de la economía agraria y los aportes de la sociología rural y agraria tanto en Cuba como en América Latina (LEYVA, 2006, p. 35). por las transformaciones y políticas económicas y sociales implementadas o por el abundante material empírico recopilado por la ciencia, en meso y micro escalas sociales, que imposibilitan homogenizar criterios.

Los campesinos constituyen en la sociedad un grupo social diferenciado como clase social o fuerza social, como economía o como cultura. Esta diversidad de concepciones busca tipificar al campesinado, a partir del momento histórico en que se enmarca el sujeto. Esta diversidad no significa una dispersión conceptual, cada uno encierra implícitamente una serie de nociones que han variado según los contextos: economía campesina, explotación familiar, grupo social, clase social.

En el contexto particular cubano, debe ser estudiado desde una concepción abarcadora o restringida. Estas dos direcciones conllevan al dilema teórico de definir universalmente al campesinado que se manifiesta variado, heterogéneo y con diferenciaciones dentro la estructura social. También existen preocupaciones acerca de cuáles son los capitales que posee este grupo humano como clase social. En sentido general, sin ánimo de definir o conceptualizar, se hace referencia a los sujetos económicos que poseen y explotan un pedazo de tierra como la base de reproducción familiar. Estos productores como regla se ocupan de la explotación de la tierra con ayuda de su familia y poseen una cultura e identidad relacionadas con la producción agraria. 
Las investigaciones que se desarrollan acerca del medio rural muestran el cambio en el contexto y las diversas transformaciones económicosociales. Asimismo, las concepciones a las que se hace referencia reflejan implícitamente o explícitamente, nuevos indicadores de análisis desde los referentes metodológicos.

A partir de la formas de propiedad, se constata la existencia de un campesinado diverso y heterogéneo que se estructura en privado (que agrupa a pequeños productores individuales (PAI) y asociados a Cooperativas de Créditos y Servicios (CCS)); socialista cooperativo (agrupa a campesinos y trabajadores agrícolas asociados a Cooperativas de Producción Agropecuarias (CPA)).

La sociología del conocimiento es la perspectiva teórico-metodológica que permite el desarrollo de la investigación, pues posibilita establecer una relación dialéctica entre el conocimiento científico que se ha producido y su contexto social de referencia. Las referencias teóricas, conceptos asumidos o contextualizados y el análisis de la propia transformación de estos elementos-resultado de la implementación o no de alguna política o por la agencia de la propia ciencia-son indicadores que se tienen presentes para el desarrollo de los resultados.

Esta perspectiva teórico-metodológica, unida a la aplicación del análisis de contenido, muestran el desarrollo de la concepción acerca del campesinado en Cuba, resumido anteriormente. Además, permite identificar elementos necesarios para el análisis de la reproducción social de este grupo humano y los aspectos teóricos, que para el contexto cubano, pueden constituirse en los capitales presentes en la sociedad cubana en general y el espacio rural.

\section{Fundamentación teórica}

El concepto de reproducción social posee diversas acepciones y son asumidas como procesos diferentes: reproducción de clases sociales; reproducción humana; reproducción económica (reproducción del modo de producción, reproducción de la fuerza de trabajo, reproducción de las relaciones de producción); reproducción cultural (el ámbito supraestructural y más abarcador frente a la reproducción social, que se identifica como la reiteración de las clases sociales a través del tiempo), entre otras (DOWBOR, 1994; COMAS, 1998; WILLIS, 1993).

Teorías más contemporáneas acerca de la reproducción social han integrado estos posicionamientos en tres tipos: la reproducción biológica, que en el plano familiar significa tener hijos y en el plano social se refiere a los aspectos socio-demográficos de la fecundidad; la reproducción cotidiana, o sea, el mantenimiento de la población existente a partir de las tareas domésticas de subsistencia; la reproducción social, todas las tareas extraproductivas dirigidas al mantenimiento del sistema social (JELIN, 1984).

Cada una de ellas lleva a reflexionar sobre la concepción de la sociedad, no como una organización meramente reiterativa, pues existen rupturas y resistencias en la manera en que se da la continuidad de sí misma:

el modelo de reproducción social es un patrón con respecto al cual se miden los cambios registrados, para concluir que se ha permanecido o no dentro del mismo caso de referencia, que no se ha salido del tipo de estructura descrito (PASSERON, 1983, p. 433-434).

Todo proceso social de producción es al propio tiempo proceso de reproducción, en la comprensión del proceso de reproducción social hay que hacer un punto de anclaje en el proceso productivo o en la producción de la vida material como base para entender las subjetividades humanas, y en un sentido más específico, el proceso de reproducción de una clase social.

Los autores de La reproducción (BOURDIEU y PASSERON, 1979), distinguen el sentido biológico del concepto de una acción social que más allá de conservar patrones rígidos heredados, es capaz de incorporar el aprendizaje de las relaciones sociales que establece la sociedad en su funcionamiento. La cultura es un elemento fundamental, como lo es la existencia de una sociedad dividida en 
clases, para esta la reproducción se convierte en el resultado de la "socialización"4 en diferentes instituciones, con funciones específicas de perpetuar determinada cultura o ser influida por otros factores, que mutarían el proceso por el cual se mantendrían determinados patrones.

La reproducción, como proceso, no es solamente la posibilidad de repetir el modelo hegemónico, sino la posibilidad de adaptarse a nuevas condiciones sociales, y bajo los mismos mecanismos, recreary resistirla violencia simbólica impuesta por diferentes acciones pedagógicas o procesos encargados de repetir la arbitrariedad cultural, que contribuye a la reproducción de las relaciones entre los grupos o las clases. Se manifiesta como un proceso construido histórico y culturalmente por diferentes sectores sociales $\mathrm{y}$ en el que los capitales que intervienen en la interacción habitus-campo, son fundamentales.

El habitus es un principio unificador y generador de prácticas que constituyen un proceso por el que lo social se interioriza en los individuos y logra que las estructuras objetivas concuerden con las subjetivas. (BOURDIEU, 1988). El campo pasa a ser un criterio de existencia del habitus y de lo social; está constituido por dos elementos: la existencia de un capital común y la lucha por su apropiación (GARCÍA CANCLINI, 1990). El capital específico describe los bienes materiales y simbólicos acumulados a lo largo del tiempo; en

4. La socialización no es concepto abordado por Bourdieu y Passeron en esta obra, ellos abordan el de acción pedagógica: comprendida como un elemento simbólico de violencia ejercido por los miembros de un grupo familiar, o por el sistema de agentes explícitamente designados a este efecto por una institución (BOURDIEU y PASSERON, 1979). Entre ambas definiciones, se puede establecer una analogía, ya que socialización se puede entender como un proceso social de aprehensión e internalización de patrones y valores culturales, la transmisión de determinados símbolos que permiten la reproducción de la cultura y de la adaptación del individuo a la sociedad. Ambos constituyen un poder simbólico que se ejerce en una relación de comunicación sobre determinada arbitrariedad cultural: inculcar ciertos significados, tratados, patrones, valores, etc. La arbitrariedad como construcción realizada por los hombres es entendida como "la selección de significados que definen objetivamente la cultura de un grupo"; arbitrariedad en tanto no puede deducirse de la "naturaleza humana" ni de la "naturaleza de las cosas" (BOURDIEU y PASSERON, 1979, p. 21). el mismo se reproducen las relaciones de poder y de autoridad de aquellos que acumulan mayor capital, sobre aquellos que menos poseen. El campo estructura el habitus, es el soporte donde ocurren todas las relaciones, prácticas sociales y culturales. El habitus contribuye a constituir el campo como mundo significativo, dotado de sentido y de valor.

En esta relación, la adaptabilidad puede contraponerse a un modelo que arbitrariamente es construido y ejercido desde quienes ostentan el poder; pero sobre todo, la reproducción social puede mantenerse en las condiciones donde factores económicos o de producción no transformen el nivel simbólico de las clases y se constituya en prácticas establecidas para ellas, pero no legitimadas.

De la misma forma, para cada una de las premisas establecidas por Lenin (1975, p. 228) para definir una clase social ${ }^{5}$, se establecen relaciones simbólicas y culturales en función de los capitales relacionados con el campo específico donde se desarrollan las relaciones económicas. Para el caso concreto del campesinado deben exponerse elementos conceptuales para su estudio.

Los estudios acerca del campesinado se insertan en las investigaciones sobre lo rural en su amplio espectro, y en el sistema de relaciones agrarias. Como sujetos activos en la producción agrícola en la sociedad son un grupo social diferenciado, que en la literatura se clasifica como clase social o fuerza social, como economía o como cultura.

Las obras clásicas del marxismo ${ }^{6}$, que abordan el tema campesino, analizan cómo se desarrolla la

5. "son grandes grupos de hombres que se diferencian entre sí por el lugar que ocupan en un sistema de producción social históricamente determinado, por las relaciones en que se encuentran con respecto a los medios de producción, por el papel que desempeñan en la organización social del trabajo, y, por consiguiente, por el modo y la proporción en que perciben la parte de la riqueza social de que disponen" (LENIN, 1975, p. 228).

6. Para ampliar la perspectiva de Carlos Marx y Federico Engels acerca del campesinado se sugiere la lectura de las obras: El dieciocho brumario de Luis Bonaparte (1852), Prefacio a La guerra campesina en Alemania (1870), Proyecto de respuesta a la carta de V. I. Zasulich (1881), El problema campesino en Francia y Alemania (1894) etc. 
forma capitalista de producción en los escenarios rurales: "la separación radical entre el productor y los medios de producción" (ENGELS, 1974), se vincula a la distribución, concentración y apropiación de la producción y medios fundamentales, para producir la formación del capital y la estratificación social de la población campesina.

En El dieciocho brumario de Luis Bonaparte, Marx (1974) se debate en los marcos de una definición ambivalente acerca del campesinado, le retira la condición de clase. Contrario a este posicionamiento, Lenin ${ }^{7}$ no concibe al campesinado como un grupo en descomposición en el modo de producción capitalista. En sus análisis del período de tránsito al socialismo anuncia en aquellas sociedades periféricas el mantenimiento del campesinado como una clase social capaz de reproducirse, pese a los efectos de la estratificación interna, ${ }^{8}$ generada por el tipo de producción, forma de comercialización, medios en explotación, uso de fuerza de trabajo, ingresos.

Por otra parte, Alexander Chayanov (1974), seguidor de la obra de Lenin, en su obra $L a$ organización de la unidad económica campesina, elabora para el contexto ruso una construcción teórica que explica el comportamiento económico

7. Lenin aborda el tema del campesino, la cuestión agraria y la cooperativización en obras como: Nuevos cambios económicos en la vida campesina (1893), El desarrollo del capitalismo en Rusia (1899), Sobre la cooperación (1923) y Sobre el impuesto en especie $(\mathrm{s} / \mathrm{d})$.

8. Distingue tres grandes grupos que a su vez pueden tener diferencias a su interior de acuerdo con criterios ya mencionados: a) un grupo superior: constituido por los campesinos acomodados o ricos, cuyos medios de producción son considerablemente superiores al nivel medio y cuyo trabajo se distingue por su mayor productividad, son los principales productores agrícolas y predominan sobre los demás grupos; por su carácter, la hacienda de este grupo es comercial, basada en grado considerable en la explotación del trabajo asalariado (p. 65); b) Un grupo medio: grupo inestable compuesto por campesinos que viven exclusivamente de los ingresos provenientes de la tierra que ellos mismos cultivan y cubre escasamente las necesidades perentorias de la familia. Hay insuficiencia de animales de labor y aperos de labranza, así como su desigual distribución (p. 55-56); c) Un grupo inferior: su rasgo fundamental es la venta de su fuerza de trabajo y el arriendo temporal de la tierra. No siembran o siembran poco, por carecer de animales de labor y los aperos necesarios. Se convierten en braceros (LENIN, 1981, p. 45). del campesinado. Su contribución radica en exponer que, como unidad de producción, no produce para acumular sino en función de las necesidades del consumo familiar.

William I. Thomas y Florian Znaniecki (1974) en la obra The Polish Peasant (1918-1920) elaboran una construcción teórica acerca del campesinado que incluye a la familia como unidad y parte de la vida comunal, con un cierto soporte económico de continuidad en la idea de la tierra, expresión de la unidad del grupo en la vida económica.

Para Theodor Shanin (1972), la explotación campesina $^{9}$ forma una pequeña unidad de producción-consumo que encuentra su principal sustento en la agricultura y es sostenida, principalmente, por el trabajo familiar. El componente familiar obtiene relevancia, pues se le asigna el rol estructurador de la actividad agropecuaria.

Entre las concepciones del enfoque marxista ortodoxo de los estudios campesinos (PÉREZ y SEVILLA-GUZMÁN, 1976) se encuentran las de Robert Redfield y Eric Wolf. La perspectiva antropológica de estos autores percibe una dependencia en términos económicos, políticos y sociales, de la sociedad global. Determina que un agricultor rural es campesino cuando mantiene una relación de dependencia ${ }^{10}$ respecto al resto de la sociedad, a la que por supuesto, permanece integrado, en términos económicos, culturales y políticos.

Se puede generalizar que las concepciones acerca del campesinado comparten la unidad unívoca de economía, institución familiar y cultura. Cada uno de los vértices influye en el resto: como grupo social posee la raíz de su diferenciación en la posición que ocupan en las relaciones de

9. La explotación campesina es un término acuñado por Lenin (1981, p. 37).

10. La relación de dependencia se fundamenta en la concepción de Redfield y Kroeber de part-societies con part-cultures. El campesino mantiene una relación de dependencia e influencia sobre la cultura del sistema social del que forma parte, que no es autónoma y suele tener carácter económico: se crea una dependencia del mercado que entra en contradicción con el modo de producción campesino (KROEBER, 1948 apud REDFIELD, 1956). 
producción, donde la unidad familiar es la fuerza productiva insertada en la economía que establece una relación asimétrica con el resto de la sociedad.

En este sentido, el análisis acerca del campesinado implica el reconocimiento de la dependencia impuesta por la desigual distribución del poder y por el carácter de la red de relaciones sociales y su diferenciación cualitativa de orden cultural. Los tres núcleos de análisis fundamentales para las interpretaciones y explicaciones en torno a la población rural, que tiene a la producción agrícola como actividad económica fundamental, se vinculan por elementos que, a la vez, forman parte de los capitales de clase:

a) Económico: parte de la relación que se establece a partir de la forma de tenencia de la tierra y las relaciones de producción.

b) Socio-familiar: estrechamente vinculado al núcleo económico, pero distingue la división laboral a partir del sexo y grupos etarios y los aportes de cada uno de los miembros a la economía campesina.

c) Cultural: vinculado a formas superestructurales que se reproducen e inciden de manera significativa en la diferenciación interna de la clase campesina.

El campesinado cubano es una clase social, agrupa a todo propietario de tierra (propietario, copropietario) privado o cooperativo, cuyos ingresos y actividad fundamental se vinculan al trabajo directo en una unidad productiva agropecuaria. Incluye, además, a los familiares que dependen económicamente de la actividad agropecuaria y se vinculan de una forma $u$ otra al trabajo en la finca. También, aquellos usufructuarios que manifiestan un capital cultural similar al de los propietarios (Gráfico 1).

\section{Metodología}

El sociólogo español Emilio Lamo de Espinosa en La sociología del siglo XX (2001, p. 23) destaca la utilidad de la ciencia sociológica en la comprensión del pensamiento producido por sí misma. Expresa: “... al abordar el pensamiento (y, en primer lugar, el propio), no puede dejar de

Gráfico 1. Estructura interna del campesinado cubano

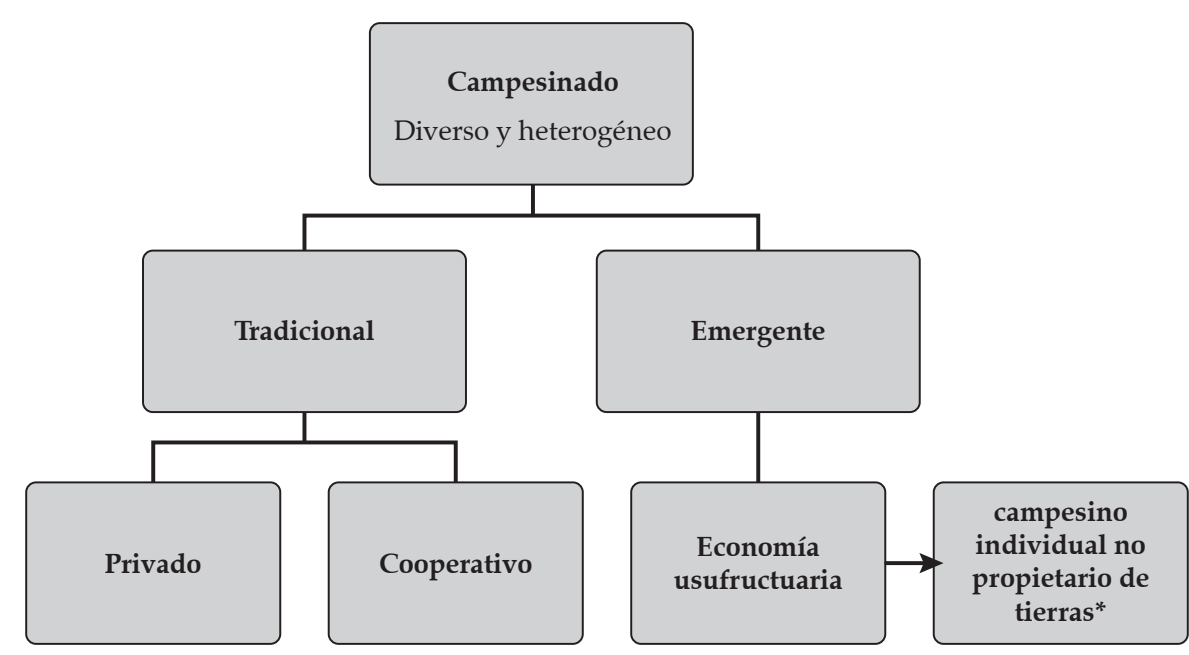

\footnotetext{
* Por su significativo peso económico, este segmento social (usufructuario) puede legar a constituir, junto con los propietarios, un componente fundamental del núcleo de los campesinos organizados en CCS. (LEYVA, 2006, p. 58)

Su constitución interna (los nuevos campesinos usufructuarios) estaría formada por agricultores que cedieron sus tierras al Estado y luego acudieron a ampliar, con la ocupación legal o precarios en los predios estatales, los minifundios que recuperaron. (LEYVA, 2006, p. 49)

Fuente: Elaboración propia a partir del análisis de contenido.
} 
indagar las condiciones sociales de su producción, es decir, no puede no hacer sociología del conocimiento $\mathrm{y}$, por lo tanto, sociología de la sociología." Esta condición le imprime un carácter reflexivo, al tratar de reconocer el contenido del pensamiento y las causas, en función de la realidad social donde se concibe el pensamiento.

La sociología del conocimiento es una especialidad sociológica que establece una perspectiva de análisis para desarrollar esta investigación, permite realizar un recorrido histórico por las ideas que constituyen los antecedentes del discurso científico y proyectarse hacia el futuro a partir del estudio de las posibles incidencias del nuevo pensamiento producido (MUÑOZ y HERNÁNDEZ, 2011).

Afirma Lamo de Espinosa (1994), que es un conocimiento de tercer nivel, un metaconocimiento, cuya reflexividad es usada sistemáticamente como método de análisis del discurso. La tradición sociológica reconoce una concepción de la sociología del conocimiento, moderada, a la que se adscribe Max Scheler y una concepción radical a la que se adscribe, Karl Mannheim. ${ }^{11}$ Desde la concepción moderada orienta el problema general para establecer hasta qué punto el pensamiento refleja los factores determinantes propuestos o es independiente de ellos. Su posición a nivel empírico lleva a investigar, lo más concienzudamente posible, las relaciones concretas entre el pensamiento y situaciones históricas (WINDELBAND y HEIMSOETH apud BERGER y LUCKMANN, 1974).

La "sociología del conocimiento es por un lado una teoría que intenta analizar la correlación que existe entre el pensamiento y la existencia, y por otro, un método de investigación histórico-sociológico que intenta descubrir las formas concretas que esta correlación ha adoptado con el desarrollo

11. La concepción moderada analiza las relaciones concretas entre el pensamiento (determinado socialmente) y sus situaciones históricas; la sociedad y los intereses sociales son identificados como elementos condicionantes del conocimiento. La concepción radical introduce la relación con el concepto general de ideología, pues el conocimiento solo puede darse desde una posición determinada (BERGER y LUCKMANN, 1974). intelectual de la humanidad" (MANNHEIM apud LAMO DE ESPINOSA, 1994, p. 14).

El proceso de producción de conocimientos científicos es una actividad de reconstrucción analítica y de creación, no es aislada, ni reproductiva; su despliegue y capacidad transformadora están sujetos a condicionantes sociales. Esta reconstrucción sobreviene a partir de la relación que se establece entre teoría y empiria, que se origina en la teoría del conocimiento sociológico.

El campo científico de la sociología rural se convierte en elemento de análisis en esta investigación mediante el análisis devarias fuentes secundarias que permiten llegar a los resultados. Tiene como referente las producciones científicas cubanas desarrolladas desde la academia: libros publicados, ponencias de congresos, tesis defendidas, artículos de revistas científicas. El análisis de contenido se convierte en una técnica necesaria para identificar el centro de interés: el contexto en el que se realizan las investigaciones y el acercamiento a los capitales que intervienen en la reproducción social del campesinado (económico, cultural, social y simbólico).

\section{Resultados y discusión}

\subsection{Contexto y producción científica en el campo de los estudios rurales en Cuba}

Los estudios en los espacios rurales antes del período revolucionario llevan a dar una mirada al motor de desarrollo, la economía agraria monoproductora y monoexportadora de azúcar de caña. Con el triunfo revolucionario de 1959, las transformaciones económicas, políticas y sociales, se encaminan a modificar una problemática social expuesta en 1953 en La historia me absolverá (alegato de autodefensa de Fidel Castro en el juicio a los asaltantes al Cuartel Moncada), desde la crítica marxista se valora histórico-socialmente la sociedad cubana. En este documento se presenta un análisis agrario y de la estructura social rural, es reconocida la situación de la unidad familiar 
campesina y la necesidad de la socialización de la propiedad de la tierra.

En Cuba la agricultura tiene presencia desde la etapa precolombina, pero es con la colonización española que se generaliza como actividad económica para el sustento de la población. A pesar de las excelentes condiciones para el cultivo de la caña de azúcar, se mantuvo al margen de la economía de plantación hasta mediados del siglo XVIII ${ }^{12}$. A razón, Manuel Moreno Fraginals (1978, p. 15) señala: "Cuba poseía, en grado superlativo, las cuatro condiciones objetivas fundamentales requeridas en el siglo XVIII para asentar una gran manufactura azucarera." ${ }^{13}$

La sociedad esclavista manifiesta la concentración, en pocos, de la propiedad de la tierra y la explotación de mano de obra en condiciones de esclavitud: el hecho de que Cuba se inserte en el mercado mundial capitalista con el azúcar, es fundamental en el auge y consolidación de la misma. En este período histórico comienzan a manifestarse formas capitalistas de explotación, al iniciar la inversión norteamericana en diversos sectores de la agroindustria azucarera y en la ganadería.

La etapa republicana comienza en 1902 bajo los influjos de una crisis en el escenario rural debido a la destrucción y despoblamiento, luego de la guerra de independencia. La estructura de tenencia de la tierra es dominada por el latifundio y campesinos minifundistas, campesinos sin tierra y asalariados agrícolas es expresión de relaciones

12. El cultivo de esta gramínea se extendió a España y Santo Domingo a inicios de los siglos XV y XVI, respectivamente, y con posterioridad se introdujo en Cuba.

13. "A saber: Primero: tierras fértiles, de fácil explotación, situadas cerca de la costa, con fácil acceso a los puertos de embarque. Segundo: bosques que proporcionasen maderas de gran calidad para la construcción de trapiches, carretas e implementos, y para levantar el conjunto de edificios requeridos, aparte de suministrar combustible (leña) durante toda la zafra. Tercero: ganado abundante que alimentase a los esclavos y tirase del trapiche y las carretas. Por lo general, el buey era la única fuerza motriz del ingenio. Excepcionalmente se utilizó el mulo o la fuerza hidráulica en el trapiche, pero el transporte de cañas y azúcares quedó siempre a cargo de los bueyes. Cuarto: Instrumentos de trabajo" (MORENO, 1978, p. 15). capitalistas de producción y distribución con formas precapitalistas de explotación ${ }^{14}$.

El problema agrario se convierte en objeto de atención por parte de varias ciencias: la historia, la sociología, la economía, la filosofía, el derecho y otras. La misma trasciende al pensamiento intelectual de Fernando Ortiz y de Carlos Rafael Rodríguez; en lo político, origina posturas críticas desde los primeros años de la república neocolonial en figuras como Manuel Sanguily, Carlos Baliño, Julio Antonio Mella y Antonio Guiteras. Sin embargo, fueron los académicos norteamericanos los precursores de las investigaciones sociológicas rurales de Cuba. Entre estas se encuentran: Problemas de la nueva Cuba; El censo agrícola de 1946; Rural Cuba, de Lowry Nelson (1951); Estudio sobre los trabajadores agrícolas cubanos (1956-1957).

Con el triunfo de la Revolución y en el contexto de la nacionalización y la Reforma Agraria, grupos de intelectuales a favor del nuevo sistema político, dan seguimiento a las principales transformaciones. La ciencia se adapta, y en especial la sociología, comienza a dar miradas hacia las clases y grupos sociales con un enfoque desde la teoría marxista-leninista.

De manera sintética, el período revolucionario en sus primeras décadas no fue de amplias producciones científicas, se implementan las transformaciones radicales en todas las esferas de la vida social, política y económica de la sociedad cubana. Los estudios evalúan el impacto de las transformaciones: cambios socioestructurales y del cuadro clasista, análisis de la composición de los sectores sociales, elevación de la instrucción y diversificación de rasgos socioprofesionales y el desarrollo del cooperativismo.

En el período de los años sesenta a los ochenta, Pavó (2012) distingue claramente dos etapas: una primera de los años sesenta hasta 1970, en la cual la literatura agrarista producida enfatiza el tema de la Reforma Agraria y sus impactos en el escenario rural. Otra, iniciada a finales de los setenta y

14. El Informe de la Foreign Policy Association (1935), titulado Problemas de la Nueva Cuba expone el descenso de los sitios de labor de 60711 en 1899 a 38405 en 1933. 
que continúa en los ochenta, centra la atención en el tema de la cooperativización desde: a) una perspectiva teórica que sustenta desde lo político y económico, la implementación en el contexto cubano, y b) contrastando empíricamente en la realidad, evaluando aciertos y dificultades.

El interés por teorizar y evaluar el comportamiento de las clases sociales y los actores rurales se despierta a medida que se transforma la nueva sociedad. Como tendencia en este período se valora una estructura de homogeneización social, es decir, desaparecen los contrastes sociales más fuertes, se produce una mayor aproximación entre grupos y clases, que presentan una situación económica menos diferenciada.

En esta situación influye, además, la implementación de una política social basada en la igualdad y la justicia social. La fundamentación desde el marxismo-leninismo, se presenta incompleta: la idea de la homogeneidad social prevalece y las políticas de gobierno se orientan hacia la igualdad de derechos y la justicia social, pero no se reconocía la complejidad de las contradicciones y las desigualdades que presentaba una sociedad que no deja de ser heterogénea.

Esta etapa se destaca también por los primeros análisis sociológicos desarrollados por investigadores cubanos sobre la estructura social rural, con el desarrollo de una perspectiva teóricometodológica propia. Los estudios sociológicos acerca de los campesinos se desarrollan en una relación estrecha entre la investigación científica y la formación académica.

La formación académica se fundamenta en planes de estudios, en estos, las asignaturas combinan el pensamiento social cubano, la orientación crítica latinoamericana, la sociología norteamericana del estructural-funcionalismo y en los setenta, se extiende la teoría marxista-leninista, como principal fuente teórica (NUÑEZ, 1997). Luego, al desarrollarse el intercambio con los países del campo socialista, las relaciones académicas se amplían al estimular y regular las líneas teóricometodológicas seguidas por los investigadores cubanos: se generaliza la concepción de la sociedad sin clases; los argumentos y tesis establecidos desconocen muchas de las contradicciones particulares de la sociedad cubana en el tránsito hacia el socialismo, en especial, las complejidades de los escenarios rurales.

Se destacan las siguientes contribuciones:

- El campesinado, desde el análisis del contexto histórico marcado por las relaciones precapitalistas de explotación existentes en el período de la república neocolonial, es caracterizado a partir de la tenencia o no de la propiedad de la tierra. Se identifica a la burguesía agraria o latifundista; campesinos medios y campesinos pobres, que en su mayoría no eran tenentes de tierra y hacían uso de ella mediante el arrendamiento, la aparcería y el precarismo.

- Los estudios sociológicos en los primeros años del período revolucionario, especialmente, los realizados en función de la estructura socio-clasista, reconocen la existencia de grupos de campesinos diferenciados en ricos, medios y pobres. Las mismas siguen los preceptos teóricos planteados desde el pensamiento social cubano de Blas Roca, Antero Regalado y Carlos Rafael Rodríguez y reflejan el pensamiento marxista-leninista al considerar al campesino como clase social heterogénea y dividida internamente.

- Respecto a los estudios del proceso de cooperativización aparece el campesinado cooperativista como grupo de la estructura socio-clasista en las formas de propiedad y producción socialista. Hay una catalogación de campesino trabajador al destacar el rol familiar en la producción agropecuaria. Es importante señalar que se reconoce, desde la teoría económica y política, la forma cooperativa como superior, pero contradictoriamente, el elemento económico incide en el reconocimiento y adhesión al grupo de cooperativistas.

Estas contribuciones son frutos de los estudios de Iliana Rojas Requena quien inicia esta mirada hacia la estructura de la sociedad cubana. En 
Algunos problemas acerca de la estructura socioclasista (s/d.), se compilan estudios sobre estructura social y la eliminación de las diferencias de clases desde la concepción euro-oriental. Su contribución continúa en otros períodos en los que la indagación empírica es focalizada hacia la relación reproducción-desigualdades sociales.

Se perciben nuevos temas de debate a partir de los procesos de cooperativización y urbanización emprendidos desde la segunda mitad de los setenta. Se evalúan los procesos agrarios, socioclasistas y sociocomunitarios, así como el modo de vida campesino en las primeras etapas de la Revolución en Estructura social y transformaciones agrarias en Cuba (RAVENET y HERNÁNDEZ, 1984).

A inicios de los ochenta, surge el Equipo de Estructura Social, perteneciente al Centro de Investigaciones Psicológicas y Sociológicas (CIPS), adscrito a la Academia de Ciencias de Cuba. Este, a partir de las demandas de investigación, comienza una sistematización y profundización de las dinámicas socioestructurales: movilidad, reproducción y el constante monitoreo sobre los cambios y tendencias que se producen en la composición socioclasista como resultado de la dinámica socioeconómica.

Entre sus estudios, los rurales privilegian al campesinado debido a la dinámica reproductiva experimentada como efecto de los procesos agrarios, entre ellos, la cooperativización agrícola y de desarrollo rural, implementados centralmente. Aunque el centro de atención gira en torno a la dinámica reproductiva, los estudios carecen de análisis integrales, pues se centran más en la movilidad apreciada, y en develar los elementos subjetivos, sin establecer la explicación de la determinante económica que acompaña al capital cultural y simbólico de las clases sociales. La complejidad social impide abordar las diferencias territoriales de las escalas micro ${ }^{15}$.

15. Al respecto vale citar informes del Equipo de Estructura Social del CIPS: El desarrollo de la cooperativización en Cuba: atraso en el inicio y situación actual (1984); El campesinado en Cuba. Estratificación interna y procesos fundamentales (1991). También la publicación del Anuario de la sociedad cubana contemporánea (1988) que recopila varias de las investiga-
Una nueva etapa comienza con el derrumbe del campo socialista: el Período Especial $^{16}$ y la implementación de la reforma económica. El surgimiento de las Unidades Básicas de Producción Cooperativa (UBPC) determina un proceso de constante evaluación para la nueva forma de organización de la producción, una vez, que las antiguas formas, (Cooperativa de Créditos y Servicios (CCS), Cooperativa de Producción Agropecuaria (CPA) y empresa estatal) sirven de patrón de comparación. Se reconstruye el aparato teórico-conceptual en virtud de adaptarlo a una realidad distinta, más diferenciada y compleja, donde los esfuerzos investigativos requieren de la conjugación de varias disciplinas desde la visión holística.

El tema de la reproducción socioclasista, objeto de investigación con ciertos resultados de investigación en el país, aflora como un fenómeno indispensable en el conocimiento científico; continúa la tendencia iniciada por el grupo de estructura social del Centro de Investigaciones Psicológicas y Sociológicas (CIPS) de relacionar estos con los de movilidad. La estructura social en este contexto se modifica en la medida en que cede paso a otra nueva ${ }^{17}$. Es meritorio destacar la

ciones realizadas sobre la estructura socioclasista en el escenario rural.

16. Es un concepto político- económico de sobrevivencia en tiempos de paz, que expresa la disposición para combatir la crisis económica con el esfuerzo y energía propios del país, para afrontar las difíciles circunstancias y hallar alternativas eficaces de solución, sin traicionar el socialismo. Es resultado de situaciones acumuladas y agravadas entre la década de los setenta y los noventa del Siglo XX en Cuba. Entre las causas directas está derrumbe del socialismo euro Soviético al que se añade el recrudecimiento del bloqueo de los Estados Unidos con la aprobación de la Ley Torricelli (1992) y la Ley Helms-Burton (1996). Su manifestación fue la crisis económica de los noventa: la pérdida de los mercados y de los vínculos económicos que se habían forjado dentro del CAME; los abastecimientos de alimentos, tecnologías e insumos y se eliminaron o disminuyeron de manera violenta y lo mismo sucedió con el financiamiento externo y los recursos energéticos (ECURED, 2015).

17. La fuerte modificación que la reforma produce en la composición socioestructural cubana da paso a una nueva etapa en el proceso de reproducción de la estructura social cubana que puede ser denominada como de "reestratificación". Es un proceso que altera cuantitativa y cualitativamente las relaciones sociales: hacen apare- 
Tesis en Opción al Grado Científico de Doctor en Ciencias Sociológicas: Reproducción socioclasista en Cuba, de Mayra Espina Prieto (1993).

Resaltan resultados científicos fundamentalmente en torno a los siguientes temas: el reordenamiento agropecuario, formas de organización de la producción agropecuaria; el impacto de las transformaciones agrarias desde el orden económico y sociológico; los cambios tecnológicos en la agricultura y las expresiones territoriales de la estructura socioclasista (estratificación, reestratificación, grupos en la estructura agraria, en función de división interna y papel de la mujer).

La producción científica no se realiza de forma aislada. La formación académica de profesionales en sociología y otras disciplinas afines, así como en otras especialidades, permiten la conformación de grupos de investigadores interesados en los estudios rurales. Estos se distribuyen a lo largo de la geografía cubana, aunque la mayoría se localiza en la capital del país y otros de igual relevancia se organizan en otras universidades.

Las producidas desde el paradigma de la economía política, analizan formas cooperativas en la agricultura: rentabilidad, productividad del trabajo, introducción de prácticas agroecológicas mediantela ciencia y la técnica. Consecuentemente, aún cuando el análisis sociológico es insuficiente,

cer nuevos estratos, transforman la situación de otros, cambian la jerarquía entre ellos. Se perciben tendencias socioestructurales vinculadas por un lado, la aparición de nuevas formaciones de clases y por otro, la fragmentación interior de los grandes componentes socioclasistas precedentes (reestratificaciónintraclasista), que se expresa en la división entre ocupados en diferentes sectores de propiedad y entre ocupados en sectores tradicionales y emergentes; la potenciación de la diversificación profesional y el reordenamiento de la producción agropecuaria a través de la parcelación y cooperativización de tierras estatales, y potenciamiento de la pequeña propiedad y la introducción de mecanismos de mercado ha implicado, por una parte, la emergencia de nuevos grupos sociales (cooperativistas en tierra del estado o UBPC y parceleros), produciéndose un verdadero proceso de "recampesinización" del agro cubano. ESPINA, M. Panorama de los efectos de la reforma sobre la estructura social cubana: grupos tradicionales y emergentes. In: CONGRESO DEL LATIN AMERICAN STUDIES ASSOCIATION (LASA), 21, 1998, Chicago. Disponível em: <http://biblioteca.clacso.edu.ar/ ar/libros/lasa98/EspinaPrieto.pdf $>$. se destacan acercamientos a la incidencia de estos factores en los movimientos ocurridos al interior del grupo social de los campesinos en la economía cubana desde 1959, la cultura de trabajo campesina y la identidad cooperativa. Los investigadores concluyen con estas consideraciones:

- Las reformas agrarias propician cambios en la estructura agraria y en las relaciones sociales de quienes las llevan a cabo.

- Se reconoce la heterogeneidad de la economía de la transición del capitalismo al socialismo: las modificaciones de la estructura económico-social signan las diferentes etapas y momentos del período de transición e indican las características, evolución y tendencia de las clases y grupos sociales.

- Los cambios estructurales y funcionales adoptados originan transformaciones esenciales en la estructura socioeconómica del país, convirtiendo su economía, en una economía mixta.

- El fortalecimiento del sector campesino, en el último período de transformaciones en la agricultura, tiene como fundamento huellas de orden de conciencia social campesina en los individuos que habían emigrado a otros componentes de la estructura social, es decir, no solo por las nuevas condiciones materiales de existencia de la sociedad cubana, sino también por las de orden subjetivo no olvidadas y que prevalecían en los que retornaban.

- Se considera el proceso de movilidad como un proceso regresivo ${ }^{18}$. Le conceden la explicación de que tales movimientos en este período solo habían alcanzado una supeditación formal a nuevas formas de producción y no real (FIGUERAS, 1999).

18. Desde formas más avanzadas: obrero agrícola e industrial y/o profesional, propias del sector socialista de economía; a menos como es la campesina, que tiene sus origines en la economía feudal y edificado bajo el impacto de las relaciones capitalistas de economía subdesarrollada. 
Los estudios producidos acerca del medio rural ponen de relieve el interés de la ciencia por conocer, analizar y proponer alternativas (en los casos necesarios) en relación con las transformaciones que se implementan como políticas de gobierno. Los temas abordan principalmente, aspectos del proceso de socialización y colectivización de la tierra, sobre la estructura y reproducción socioclasista, los procesos económicos en formas variadas de organización de la producción. También se reconoce la existencia de grupos de campesinos diferenciados en ricos, medios y pobres.

El tratamiento al campesinado se presenta mediante investigaciones aplicadas y los análisis teóricos existentes, resultan insuficientes en cantidad y en integralidad, dado el desarrollo de la ciencia y la prevalencia de la visión holística propia del marxismo-leninismo. No obstante, con relación a la sociología, los diferentes conceptos y teorías asumidos se contextualizan, pues dependen de la periodización histórica y las transformaciones introducidas para el desarrollo de la agricultura y la sociedad rural.

Elabordajedelcampesinado(unidadeconómica familiar) en las ciencias sociales cubanas como objeto de estudio constituye un área abordada, y con resultados desde varias disciplinas (economía, derecho, historia, sociología y en menor medida, la antropología); pero los estudios que hacen referencia a la reproducción social -como proceso sociológico- aún son insuficientes y se vinculan a los estudios de movilidad social. Se desarrollan principalmente a niveles macroestructurales, pero no especifican las diferencias territoriales y las particularidades de cada uno de los grupos sociales, además, falta la referencia a los capitales que intervienen en la reproducción social.

\subsection{La reproducción social: los capitales que intervienen en proceso social de reproducción del campesinado cubano}

La reproducción social se manifiesta como un proceso construido, histórico y culturalmente, de percepciones colectivas que tipifican determinado patrón de comportamiento, adecuado a las condiciones sociales de las que son producto e imprimen simbolismo y definición a las funciones que van a cumplir como resultado de la interacción del nivel económico con el simbólico. De esta manera, para estudiar la del campesinado como clase social, se precisa entender y establecer su participación en las relaciones de producción e identificar las prácticas asociadas, que son más que rasgos complementarios o consecuencias secundarias de su ubicación en el proceso productivo.

Las prácticas asociadas a las relaciones de producción componen un conjunto de características auxiliares que, a modo de exigencias tácitas, pueden funcionar como principios de selección o de exclusión reales, sin ser jamás formalmente enunciadas (BOURDIEU, 1988). Su expresión lo constituye el capital específico, formado por bienes materiales y simbólicos acumulado a lo largo del tiempo y poseedor de una estructura de distribución que se relaciona con los mecanismos de reproducción. Bourdieu (1986) determina que entre las diferentes especies de capital se encuentran las siguientes: el económico, el cultural, el social, y el simbólico. ${ }^{19}$

A continuación se exponen los resultados del análisis de contenido a la producción científica realizada en Cuba sobre el medio rural, que constituyen referencias para los estudios sobre el campesinado y su reproducción social. Estas consideraciones no hacen referencia al capital simbólico, a modo de conclusión se aborda este último, pues su manifestación se relaciona con el grado mayor o menor de asimilación de las características asociadas a los otros capitales.

19. “El capital económico es entendido como cualquier tipo de bien directamente convertible en dinero; también institucionalizado en la forma de derechos de propiedad; el cultural, que puede existir en tres estados: incorporado (disposiciones, habilidades y capacidades del cuerpo y de la mente), objetivado (bienes culturales) e institucionalizado (títulos académicos); el social, entendido como la capacidad de los agentes de movilizar recursos a partir de su red de relaciones sociales y el simbólico, comúnmente llamado prestigio, reputación o renombre" (BOURDIEU, 1986, p. 243). 


\subsection{Sobre el capital económico}

Con el desmantelamiento de la estructura inherente al capitalismo dependiente cubano y la formación de una nueva estructura, se supera la propiedad privada de los medios de producción y la formación de un sistema único de economía ${ }^{20}$ (CONGRESO DEL PARTIDO COMUNISTA DE CUBA, 1975).

La implementación de diversas políticas dirigidas hacia la socialización estatal de la finca a finales de los años sesenta conduce a declinar, en número y área, el sector campesino. La vía cooperativa $^{21}$ sustituye la política de estatización y de creación de grandes planes productivos y comienza la integración de pequeños y medianos productores a las CPA. Los nuevos componentes socioestructurales se reproducen cuantitativamente de forma estable y los cambios más intensos se trasladan a nivel de la estructura interna, intentando adecuar su estructura profesional y de calificación a los diversos perfiles ocupacionales que habían emergido (ESPINA, 1997).

A instancias de estos acontecimientos, Figueroa (2005) expresa que hay una reducción en número y área del sector campesino- entendido como aquel pequeño agricultor individual o pequeño propietario. Con el salto de propietario privado a cooperativista y al incorporarse un tercio del total de propietarios privados a esta nueva forma de organización de la producción se

20. En la producción agropecuaria la forma superior de propiedad socialista es la granja estatal; la cooperativa será una forma de propiedad colectiva que supera la propiedad individual: ambas constituyen formas socialistas de producción con arreglo al Sistema de Dirección y Planificación de la Economía y con la introducción de la técnica, el uso eficiente de la tierra (principal recurso natural), los recursos materiales y humanos. Estas formas superiores de producción no son solo una necesidad económica sino una necesidad social.

21. El Capítulo V "De la Cooperación Agraria" de la Primera Ley de Reforma Agraria establece el fomento de cooperativas agrarias y otras formas de cooperación con fines de proveer recursos materiales, medios de trabajo, crédito, etc. A razón ya existían las Cooperativas de Créditos y Servicios y Cooperativas Cañeras. El I Congreso del Partido Comunista de Cuba sienta las bases para el desarrollo de las mismas. perpetúan formas de tenencia de la tierra como la aparcería y el precarismo, señales inequívocas de desajustes en el agro, que aún prevalecen.

La agricultura estatal se concentra en producir materias primas para las industrias de productos exportables, con la reducción de áreas agrícolas para otros fines. Para los cultivos con alto grado de especialización como el tabaco, el café y el cacao, se fundamentan nuevas bases jurídicas, que inciden en la estimulación a la fuerza laboral mediante la entrega de tierras en usufructo, la legalización de las distribuciones y el reconocimiento de que la producción individual o familiar es la forma de producción más eficiente en las condiciones de estos cultivos (VALDÉS, 2005).

El fraccionamiento de la gran propiedad estatal en unidades más pequeñas: (Unidades Básicas de Producción Cooperativa) ${ }^{22}$, la existencia de asociaciones con capital extranjero y el reparto de tierras que permanecían ociosas a los interesados en hacerlas producir en régimen de usufructo, se diversifica el régimen de tenencia de la tierra, da lugar a un sistema de economía mixta que tiene por pilares básicos: la economía de tipo campesino parcelero y la de base colectivo-cooperativista (FIGUEROA, 1995).

A partir del año 2005, se implementan medidas encaminadas a la búsqueda de soluciones para lograr la reactivación del sector agropecuario. El Ministerio de la Agricultura desarrolla un proceso de perfeccionamiento y reordenamiento del sistema de agricultura "que abre una nueva etapa sobre la explotación y gestión de las tierras agrícolas, considerada por algunos especialistas como la "Cuarta Reforma Agraria". La medida más importante ha sido la entrega de tierras

22. Con el objetivo de reestructurar el sector agropecuario estatal que poseía más del $80 \%$ de las tierras del país se crea una nueva estructura que tiene como principios la vinculación del hombre al área como forma de estimulación al trabajo; el autoabastecimiento de los obreros y familiares con esfuerzo cooperado; asociar los ingresos a la producción alcanzada y desarrollar la autonomía de gestión. Ver Decreto Ley número 142/93 Sobre las Unidades Básicas de Producción Cooperativa. En 2012 entra en vigor la Resolución 574/12 Reglamento general de las Unidades Básicas de Producción Cooperativa. 
agrícolas ociosas en usufructo $^{23}$ las formas que registran una mayor eficiencia son las CCS y el privado $^{24}$ (NOVA, 2013).

Como pequeña empresa económica, la unidad económica familiar campesina da valor a la tierra como medio de producción y en este proceso genera las dinámicas internas y exclusivas de la clase social (dadas las relaciones económicas vinculadas a la actividad agropecuaria y el tipo de propiedad). El uso y tenencia de la tierra condiciona en primera instancia el capital económico, el resto de los bienes se adquieren a partir de la capacidad productiva que tienen los campesinos, las formas de propiedad de los medios de producción y de distribución de la ganancia.

\subsection{Sobre el capital cultural}

Cuba exhibe avances significativos en el ámbito de la educación y en el orden social mantiene diferencias de orden cualitativo en el espacio rural (AGÜERO, 2006, p. 262):

- En estos espacios hay un incremento de la escolarización, de 3,3 grados promedio en 1953, a 7,9 grados en 1994 y esta última cifra es estable y condicionada por otros factores.

- Los intereses del sistema educativo determinan que el modelo de hombre que la escuela forma sea lo más integral posible en su formación. Interesa que no solo los actores sociales y grupos de la estructura socioclasista asciendan y se reproduzcan, sino que avancen realmente tanto en

23. Conduce a un nuevo modelo agrícola que consolida el predominio de los productores no estatales, particularmente las CCS y el productor privado, pasando de un 18,5 al 35,8 \% de tenencia de la tierra (NOVA, 2013).

24 . Estas dos formas producen el $57 \%$ de la producción total de alimentos del país, con tan solo $24.4 \%$ de la tierra cultivable, registran el 3.7 y $1.7 \%$ respectivamente de la tierra reportada como ociosa. Producen el 56\% de leche (el estado produce el 15\%), dispone de más del $55 \%$ de la vacas en ordeño y de más del $50 \%$ del ganado vacuno existente y poseen el 59\% del ganado porcino. No se dispone de estadísticas oficiales publicadas sobre los resultados económicos de las CCS y privad o, pero es de suponer por sus resultados productivos sean los más eficientes (NOVA, 2013). sentido vertical como horizontal, hacia una profesionalización, manteniendo el estatus de obrero o, pasando a otro grupo, a otra clase o capa como la intelectualidad, por ejemplo.

- La condición de una sociedad rural, que es agrícola pero no campesina, produce varios impactos en la estructura social clasista: envejecimiento de la fuerza de trabajo agrícola y lento relevo generacional.

Además, este capital se manifiesta por la cultura del trabajo campesino, la creación y el uso de determinados instrumentos de trabajo y las tradiciones productivas. En relación con la producción se perciben indistintamente conocimientos generados desde el conocimiento tradicional, dado por la socialización familiar y procesos de innovación internos; desde el conocimiento científico generado desde el extensionismo rural, o desde la integración de ambos. En el caso de los cultivos especializados su producción se basa en cánones tradicionales, sin embargo, el uso de nuevas tecnologías genera un impacto positivo en el rendimiento productivo (MACHADO, 2013).

La actividad es el proceso fundamental donde se modelan los significados y las relaciones sociales y por tanto, se adhiere al componente cultural, se dificulta la comprensión de que "La práctica productiva tecnológica es una actividad de interacción social, de transformación, cognición y valoración, en ella no solo se crea lo material sino que se utilizan y desarrollan conocimientos, experiencias y hábitos" (MACHADO, 2013, p. 9).

El uso de conocimiento tradicional y prácticas agroecológicas,cobraimportanciaporcaracterísticas económicas presentes que imposibilitan el desarrollo extensivo de la agricultura mecanizada durante el Período Especial. Casi paralelamente se abre al debate internacional de la sostenibilidad del desarrollo y su emergencia para las ciencias sociales lo que legitima la cultura campesina cubana.

Otra de las determinantes culturales se relaciona con la reproducción de la fuerza de trabajo y puede ser determinada en una doble 
dirección: una en la que las figuras masculinas y de mayor edad son portadoras de esos valores culturales tradicionales que se encuentran en peligro de desaparición por el envejecimiento de los campesinos individuales. Otra, en la que la familia campesina tradicional se plantea la estrategia de dejar a uno de los hijos en la finca.

Desde otro punto de vista, las estructuras productivas basadas en una economía con una cultura tradicional asociada a la labor agrícola y apego a la tierra, por lo general tienen más posibilidades de lograr productividad, estabilidad de fuerza laboral y consolidar un sistema de relaciones económicas como forma de organización de la producción agropecuaria. La cooperación y participación en función del uso y beneficio colectivo genera cambios en las relaciones económicas, pero no son suficientes para incorporar modelos culturales que transformen radicalmente a los individuos.

Por tal razón, la actividad productiva realizada por la unidad económica familiar es el proceso en el que se modelan los significados y las relaciones sociales en función de conocimientos, experiencias y hábitos; es fuente de valores y símbolos de la cultura. Estos últimos condicionan los saberes tradicionales generalizados como prácticas agroecológicas, la incorporación o no a cooperativas como forma socialista superior de la economía agropecuaria, los mecanismos de participación y promoción de aquellas problemáticas objeto de análisis del núcleo cultural.

El capital cultural incide también en la legitimación o deslegitimación del nuevo segmento social emergente (usufructuarios), que por la tenencia de tierra e integración familiar a la actividad productiva agropecuaria, se integra al campesinado.

\subsection{Sobre el capital social}

Comprender la complejidad de la realidad campesina supone la complementación de los factores económicos (economía campesina) y la participación de las prácticas socioculturales como agentes que definen un modo de ser ante lo identitario, histórico y social. El capital social engloba la capacidad organizativa de los campesinos para involucrar a la familia en la actividad productiva y reproductiva; así como de establecer una red de relaciones sociales.

En la unidad campesina de explotación doméstica, “...la familia debe ser reconceptualizada como un espacio en el que potencialmente convergen múltiples relaciones de clase" (DE ARMAS, 1997, p. 54). En el contexto donde se percibe a la mujer en desventaja, se ubica a la familia como gestora de relaciones sociales de múltiple carácter. El comportamiento familiar, vinculado a la producción y en función de la división social y sexual, se evalúa de acuerdo con la asociación a formas de organización de la producción y tipo de propiedad, que le atribuye características como capa de la estructura interna de la clase social.

La familia campesina desempeña el rol educativo, es gestora de relaciones sociales en función de la división sexual y social del trabajo y consecuentemente, reproduce valores y cultura. En ella, las mujeres tienen un peso importante en la decisión de innovaciones y tecnologías a implementar y en la generación de beneficios económicos derivados de las nuevas alternativas. Los ancianos son fuente de saber y ejercen poder sobre el resto de la familia.

La familia en la sociedad rural juega un
tradicional papel de pequeña empresa
económica, donde la experiencia y el
saber aplicado, verificado, acumulado y
generalizado de los campesinos lo tienen los
ancianos; y la sostenibilidad de la equidad
de género se logra considerando las edades
más tempranas: aunque en la actualidad, la
juventud rural se encuentra entre diversas
visiones que pueden negar o no la continuidad
del devenir histórico familiar y desentenderse
o no de las actividades agrícolas (MARTínEZ,
2009, p. 35).

Las relaciones que se establecen al interior del grupo familiar, son significativas para describir los roles asignados y asumidos por los miembros 
en el ámbito doméstico y en el espacio público (relaciones en función de la producción). Cada uno de los miembros que la constituyen, son portadores de prácticas, valores, significado y significante de la tierra como medio de producción, por lo que Martínez (2009, p. 36) la considera "el agente estabilizador por excelencia del sistema productivo del medio rural".

La familia campesina establece relaciones con el conjunto de instituciones y organizaciones surgidas en los espacios rurales como parte de las políticas públicas, que fortalecen la infraestructura con vías de comunicación, establecimientos comerciales, servicios educacionales, recreativos y de salud. En las comunidades rurales campesinas o tradicionales y en las inducidas- formadas a partir del proceso de cooperativización- se desarrollan los vínculos familiares y sociales en torno a los vínculos económicos y culturales.

El tránsito a las comunidades rurales inducidas, "transforma las condiciones de aislamiento y el bajo grado de interrelación social que frena el desarrollo de las fuerzas productivas" (RAVENET y HERNÁNDEZ, 1984, p. 85). Entre los vínculos económicos destacan el aumento de la cooperación interproductiva que se establece con otros tipos de economía en el espacio rural; con las empresas agrícolas y científicas encargadas de introducir los adelantos de la ciencia y la técnica. La forma de organización productiva familiar soporta formas más específicas de la superestructura como son las festividades vinculadas al inicio y fin del ciclo productivo, se generalizan dietas alimenticias, hábitos cotidianos y creencias religiosas. También aumentan los vínculos sociales formalizados; su participación en las decisiones y problemas comunales y los relacionados con la producción (DONÉSTEVEZ, FAJARDO y FIGUERAS, 1998).

El concepto de pluriactividad ${ }^{25}$ toma relevancia una vez que vincula a actores económicos no

25. El término parte del debate por la existencia de agricultura en tiempo parcial, empleos múltiples y a fines de los años '80 se amplía con destino a incluir actividades laborales no necesariamente remuneradas en dinero. De acuerdo con Fuller (1990, p. 367): "[...] la pluriactividad describe relacionados con la actividad agropecuaria y que pueden realizar los miembros de la familia campesina, una vez que su ocupación fundamental no es el trabajo agrícola y se incorporan a la actividad agrícola de manera no formal.

Relacionado a este aspecto se modifica el status de la mujer durante el crecimiento de los hijos y se percibe en la socialización de los mismos, lo que facilita la adquisición de poder a la unidad económica familiar. Asimismo, resultados empíricos constatados muestran que las mujeres tienen un peso importante en la decisión de "cuál o cuáles variedades, especies o tecnologías se evaluarán y adoptarán en las fincas. Tienen un papel importante también en la búsqueda de la generación de beneficios económicos derivados de las nuevas alternativas, ya sea por la búsqueda del valor agregado de productos conocidos o por la introducción de nuevos productos al mercado" (MARTÍNEZ, 2009, p. 55).

Otros indicadores de diferenciación interna complementan la red de relaciones sociales y constituyen parte de este capital. Tal es el caso del proceso de comercialización como fuente de ingresos superiores y el empleo de mano de obra asalariada. La venta de la producción incita a la formación de un grupo de intermediarios, dependientes del campesinado, dedicados a la comercialización de la producción y al inevitable vínculo con un sector de mercado formal o informal.

Por otra parte, la capacidad de movilizar los recursos a partir de la red de relaciones sociales, para productores individuales y campesinos cooperativistas es diferente. Las cooperativas, como sujetos jurídicos, le proporcionan a sus asociados beneficios a la hora de planificar, contratar, recibir y utilizar los recursos materiales, financieros y la asistencia técnica para lograr mayor eficiencia en los resultados de su producción; contribuir a elevar el nivel económico

una unidad productiva multidimensional, en la que se emprenden actividades agrícolas y no agrícolas a su interior y fuera del mismo y por las cuales diferentes tipos de remuneración son recibidos (rendimientos monetarios, rentas en especie y transferencias)." 
y social de sus integrantes y fomentar la ayuda mutua y otras formas de cooperación entre los agricultores y sus familiares. Esta forma de organización cuenta con el apoyo del Estado, lo que amplía la capacidad de establecer relaciones fuera de la comunidad.

En sentido general, la familia encuentra en la comunidad un territorio donde satisfacer sus necesidades económicas (materiales: infraestructura de servicios educacionales, culturales, recreativos, de salud y espirituales. La conjunción de ambos- familia y comunidadlogran la perfecta realización de las funciones correspondientes a cada una. Se distingue que la conciencia colectivista o de productor individual, repercute no solo de forma estructural en la sociedad cubana sino que sus implicaciones llegan a influir en el plano superestructural.

\section{Consideraciones finales}

La concepción acerca del campesino presentada, después del análisis de la producción científica, no es estática. Las determinantes socio-históricas influyen en su composición interna: aparición de segmentos sociales o capas y desaparición de otras. El discurso producido por la ciencia, se apropia del cúmulo de ideas precedentes y se modifica de acuerdo con los nuevos elementos que se presentan en el contexto. Una visión particularizada de los fenómenos, reconoce el desigual impacto de las políticas nacionales a nivel local que se diversifican por la forma de socialización y colectivización de la tierra, las formas de organización de la producción, características particulares de los territorios y sus determinaciones históricas.

Se denomina campesinado (unidad económica familiar) en su consideración como clase social, a todopropietariodetierra (propietario, copropietario o usufructuario) privado o cooperativo cuyos ingresos y actividad fundamental se vinculan al trabajo directo en una unidad productiva agropecuaria. Incluye, además, a los familiares que dependen económicamente de la actividad agropecuaria y se vinculan de una forma $u$ otra al trabajo en la finca, no desarrollan sistemáticamente otro tipo de actividad laboral.

El conocimiento acerca de estos sujetos activos de la producción y su perpetuación como grupo de la estructura socioclasista, permite adecuar la producción agroalimentaria a la demanda y la priorización de la sustitución de la importación, de aquellos alimentos que puedan ser producidos eficientemente en el país.

El reconocimiento, por parte de los tomadores de decisiones políticas y económicas, del incremento de la superficie de tierras productivas y volúmenes de alimentos producidos por este grupo humano, demanda a la ciencia conocer las estrategias familiares de reproducción social en unidades económicas campesinas, en función de sus capitales específicos.

Sin embargo, la carencia de este tipo de estudios en Cuba y en especial sobre los rasgos diferenciados del capital en los campesinos individuales, cooperativistas y usufructuarios, imposibilitan una adecuada formulación de políticas diferenciadas que permitan el desarrollo socioeconómico del país, en consonancia con la estructura socioclasista vinculada a la actividad agroproductiva. Los resultados que se presentan, no son concluyentes en este sentido, pero conducen a un acercamiento a los capitales con que cuenta el campesinado cubano y puede generar estrategias particulares de reproducción social.

En la reproducción social del campesino en Cuba influyen múltiples procesos, para su comprensión es necesario hacer un análisis histórico-contextual de las políticas implementadas y del grado de asimilación en las escalas sociales. Los capitales manifiestos, pueden variar de un territorio a otro, pero siempre tienen como base el capital económico, especialmente, por las formas de tenencia de la tierra (propiedad) y de organización de la producción.

Las formas de manifestación del capital social, más allá de delimitar la integración de diferentes actores, a nivel familiar, comunal o de la sociedad en general, permiten comprender que las relaciones sociales son fruto de los elementos 
Consideraciones para la Comprensión de la Reproducción Social del Campesino Cubano: acercamiento desde la producción científica rural

confluyentes en el sistema de relaciones económicas, políticas, culturales y sociales.

La familia campesina desempeña el rol educativo, es gestora de relaciones sociales en función de la división sexual y social del trabajo y consecuentemente, reproduce valores y cultura. En ella, las mujeres tienen un peso importante en la decisión de innovaciones y tecnologías a implementar y en la generación de beneficios económicos derivados de las nuevas alternativas. Los ancianos son fuente de saber y ejercen poder sobre el resto de la familia.

El capital cultural, en sentido general, establece los parámetros de éxito. El caso cubano demuestra que un índice medio de escolarización (estado institucionalizado) no es suficiente para que los resultados productivos sean satisfactorios. Es necesario, además, el saber incorporado expreso en las disposiciones, habilidades y capacidades del cuerpo y de la mente y producto de la herencia o socialización. Los bienes culturales se generan en esa interrelación permanente con el resto de los capitales.

Para determinar el capital simbólico la complejidad es mayor. Se distingue que la conciencia colectivista o de productor individual, repercute no solo de forma estructural en la sociedad cubana, sus implicaciones llegan a influir en el plano superestructural. La subjetividad de cada individuo repercute en la manera de percibirse y el prestigio se valora a partir del referente o punto de comparación. Sin embargo, para la sociedad cubana el campesinado es, entre los sujetos productores de alimentos, quien ha demostrado su capacidad de mantener su lugar en la estructura social y su creciente papel en la recuperación económica.

\section{Referencias}

AGÜERO, F. C. Sociedad, cultura y currículum escolar. Reflexiones, análisis y propuestas desde um estudio de caso en Cuba. Berlin: Wissenschaftlicher Verlag, 2005.

BERGER, P. y LUCKMANN, T. Introducción. El problema de la sociología del conocimiento. In:
(Org.). La construcción social de la realidad. Buenos Aires: Amorrortu, 1974.

BOURDIEU, P. The forms of capital. In: R. (Ed.). Handbook of theory and research for the sociology of education. s.l: Ed. Greenwood Press, 1986. p. 241-260

BOURDIEU, P. La distinction. Madrid: Taurus, 1988.

- y PASSERON, J. C. La reproducción. Elementos para una teoría del sistema de enseñanza. México: Lain S.A, 1979.

CHAYANOV, A. V. La organización económica de la unidad económica campesina. Buenos Aires: Nueva Visión, 1974.

COMAS, D. Antropología económica. Barcelona: Ariel, 1998.

CONGRESO DEL PARTIDO COMUNISTA DE CUBA, n. 1,1975, La Habana. Tesis y resoluciones: Sobre la cuestión agraria y las relaciones con el campesinado. Ciudad de La Habana: Ciencias Sociales, 1978. p. 613-670.

DE ARMAS, T. La participación femenina en el sector campesino-parcelero. Estudio de caso. 1997. 64f. Trabajo de Diploma en Sociología- Facultad de Filosofía e Historia, Universidad de La Habana, 1997.

DONÉSTEVEZ, G., FAJARDO, L. y FIGUERAS, D. Transformación agraria y desarrollo de las comunidades rurales (reflexiones para un estudio de caso) In: PEREZ, N., GONZALEZ, E. y GARCIA, M. (Org.). Campesinado y participación social. La Habana: Universidad de La Habana,1998. p. 130-140

DOWBOR, L. La reproducción social. México: Siglo XXI, 1994.

ECURED. Período especial en Cuba. Enciclopedia colaborativa cubana. Disponível em: <http://www. ecured.cu >. Acesso em: 15 jul. 2015.

ENGELS, F. Proyecto de respuesta a la carta de V. I. Zasulich. In: MARX, C. y ENGELS, F. Obras Escogidas en tres tomos, t. III. Moscú: Ed. Progreso, 1974 (p. Digitalizado para Marxists Internet Archive, 2001).

ESPINA, M. Transformaciones recientes de la estructura socioclasista cubana. Papers, s.l, n. 52, p. 83-99, 1997.

ESPINA, M. Panorama de los efectos de la reforma sobre la estructura social cubana: grupos tradicionales $\mathrm{y}$ emergentes. In: CONGRESO DEL LATIN AMERICAN STUDIES ASSOCIATION (LASA), 21, 1998, Chicago. Disponível em: <http://biblioteca.clacso.edu.ar/ar/ libros/lasa98/EspinaPrieto.pdf > . Acceso em: mar. 2010.

FIGUERAS, D. Campesino: la movilidad social y la construcción socialista en Cuba. 1999, 35, Ponencia del Grupo de Estudios de Desarrollo Rural y 
Cooperativismo, Universidad Central “Martha Abreus" de Las Villas, Villa Clara, 1999.

FIGUEROA, V. La reforma de la tenencia de la tierra en Cuba y formación de un modelo mixto de economía agraria. Santa Clara: Universidad Central de Las Villas, 1995.

. Los campesinos en el proyecto social cubano. Temas, La Habana, n. 44, p. 13-25, oct./dic. 2005.

FOREIGN POLICY ASSOCIATION. Problemas de la nueva Cuba. Informe de la Comisión de Asuntos Cubanos. New York: s.n, 1935.

FULLER, A. M. From part time farming to pluriativity: A decade of change in rural Europe. Journal of Rural Studies, v. 6, n. 4, p. 361-373, 1990.

GARCÍA CANCLINI, N. Introducción: la sociología de la cultura de Pierre Bourdieu. In: BOURDIEU, P. (Org.). Sociología y cultura. México: Editorial Grijalbo, S.A., 1990.

JELIN, E. Familia y Unidad doméstica: mundo público y vida privada. Estudios CEDES, Buenos Aires, 1984. Disponível em: <www.rau.edu.uy/fcs/dts/Mip1/ produccionreproduccion.pdf $>$. Acceso em: set. 2011.

LAMO DE ESPINOSA, E. La sociología del conocimiento y de la ciencia. Madrid: Alianza Universidad, 1994.

. La sociología del siglo XX. Reis. Revista Española de Investigaciones Sociológicas, s.l, n. 96, p. 21-49, 2001.

LENIN, V. I. Una gran iniciativa. In: (Org.). Obras Completas. T. 39. Moscú: Ed. Progreso, 1975. p. 13-26

Nuevos cambios económicos en la vida campesina. In:__ (Org.) Obras Completas. Tomo I. Moscú: Ed Progreso, 1981. p. 1-71

LEYVA, A. Cambios socioclasistas y relaciones agrarias en la provincia Granma a partir de 1993. 2006, $150 \mathrm{f}$. Tesis (Doctor en Ciencias Sociológicas) - Facultad de Filosofía e historia, Universidad de La Habana, Ciudad de La Habana, 2006.

MACHADO, Y. La práctica productiva cafetalera desde los saberes científicos y tradicionales de los productores agrícolas en Crucecitas. 2013, 91 f. Tesis (Maestría en Sociología)Facultad de Filosofía e historia, Universidad de La Habana, Ciudad de La Habana, 2013.

MARTÍNEZ, A. Innovar en la innovación. Fitomejoramiento como alternativa para promover la equidad de género en la comunidad rural Pretiles, Jibacoa. Municipio de Manicaragua, Provincia Villa Clara. 2009, 80 f. Tesis (Maestría en Sociología)- Facultad de Filosofía e historia, Universidad de La Habana, Ciudad de La Habana, 2009.
MARX, C. El dieciocho brumario de Luis Bonaparte. In: MARX, C. y ENGELS, F. Obras escogidas en tres tomos. Tomo I Moscú: Ed. Progreso, 1974. p. 404-498.

MORENO, M. El ingenio complejo económico social cubano del azúcar. Tomo 1. La Habana: Ciencias Sociales, 1978.

MUÑOZ, T. y HERNÁNDEZ, A. Para una comprensión de las raíces y el devenir. Un análisis histórico, lógico y metodológico. In: MUÑOZ, T. et al. (Org.). Para una sociología de la sociología en Cuba. Parte 1. La Habana: Universidad de La Habana, 2011.

NOVA, A. Valoración del impacto de las medidas más recientes en los resultados de la agricultura en Cuba. El Sector Agropecuario y los Lineamientos de la Política Económica Social. 2013. Disponível em: <https://www. nodo50.org/cubasigloXXI/economia/nova_311211. pdf >. Acesso em: 20 ene. 2014.

NUÑEZ, J. Aproximación a la sociología cubana. Papers, s.l, n. 52, p. 187-203, 1997.

PASSERON, J. C. La teoría de la reproducción social como una teoría del cambio: una evaluación crítica del concepto de "contradicción interna". Estudios Sociológicos, s.l, I:3, p. 425-434, 1983.

PAVÓ, R. Derecho agrario; teoría general, su recepción y estado actual en Cuba. Eumed. 2012. Disponível em: <http://www.eumed.net/libros/2012b/1213/desarrollo_ legislacion_agraria.html>. Acesso em: 14 mar. 2013.

PÉREZ, M. y SEVILLA-GUZMÁN, E. Para una definición sociológica del campesinado. Agricultura y sociedad, n. 1, s.l, p. 15-39, 1976.

RAVENET, M. y HERNÁNDEZ, J. Estructura social y transformaciones agrarias en Cuba. La Habana: Ciencias Sociales, 1984.

REDFIELD, R. Peasant and Culture. Chicago: The University of Chicago Press, 1956.

ROJAS, I. Algunos problemas acerca de la estructura socioclasista. La Habana: Universidad de La Habana, s/a.

SHANIN, T. La clase incómoda. Sociología política del campesinado en el desarrollo de la sociedad Rusa 1910-1925. Oxford: Clarendon Press, 1972.

THOMAS, W. I. y ZNANIECKI, F. The Polish Peasant in Europe and America. Tomo I. New York: Octagon Books, 1974.

VALDÉS, J. Procesos agrarios en Cuba (1959-1995). La Habana: Félix Varela, 2005.

WILLIS, P. Producción cultural no es lo mismo que reproducción cultural, que a su vez no es lo mismo que reproducción social, que tampoco es lo mismo que reproducción. In: VELASCO, H., GARCÍA, J. y DÍAZ, A. (Org.). Lecturas de antropología para educadores. Madrid: Trotta, 1993. 
